\title{
Recognition of the mycobacterial cord factor by Mincle: relevance for granuloma formation and resistance to tuberculosis
}

\author{
Roland Lang* \\ Institute of Clinical Microbiology, Immunology and Hygiene, University Hospital Erlangen, Friedrich-Alexander-University Erlangen-Nürnberg, Erlangen, Germany
}

Edited by:

Dov L. Boros, Wayne State

University School of Medicine, USA

\section{Reviewed by:}

Hiromasa Inoue, Kagoshima

University, Japan

Sho Yamasaki, Kyushu University,

Japan

\section{*Correspondence:}

Roland Lang, Institute of Clinical Microbiology, Immunology and Hygiene, University Hospital Erlangen, Friedrich-AlexanderUniversity Erlangen-Nürnberg, Wasserturmstr. 3-5, 91054 Erlangen,

Germany.

e-mail: roland.lang@uk-erlangen.de
The world's most successful intracellular bacterial pathogen, Mycobacterium tuberculosis (MTB), survives inside macrophages by blocking phagosome maturation and establishes chronic infection characterized by the formation of granulomas. Trehalose-6,6-dimycolate (TDM), the mycobacterial cord factor, is the most abundant cell wall lipid of virulent mycobacteria, is sufficient to cause granuloma formation, and has long been known to be a major virulence factor of MTB. Recently, TDM has been shown to activate the Syk-Card9 signaling pathway in macrophages through binding to the C-type lectin receptor Mincle. The Mincle-Card9 pathway is required for activation of macrophages by TDM in vitro and for granuloma formation in vivo following injection of TDM. Whether this pathway is also exploited by MTB to reprogram the macrophage into a comfortable niche has not been explored yet. Several recent studies have investigated the phenotype of Mincle-deficient mice in mycobacterial infection, yielding divergent results in terms of a role for Mincle in host resistance. Here, we review these studies, discuss possible reasons for discrepant results and highlight open questions in the role of Mincle and other C-type lectin receptors in the infection biology of MTB.

\section{Keywords: mycobacteria, cord factor, TDM, Mincle, C-type lectin receptor, tuberculosis}

\section{TDM's HISTORY AS PAMP AND GLYCOLIPID EFFECTOR MOLECULE OF MYCOBACTERIA}

Trehalose-6,6-dimycolate (TDM) has been isolated as a major glycolipid from the cell wall of pathogenic mycobacteria in the 1950s by Bloch and colleagues (see Hunter et al., 2006 for review). The biological properties of TDM have been explored in vivo using mice and rabbit models and led to the realization that TDM is sufficient to induce formation of granulomas when injected in oil doplets (Yarkoni and Rapp, 1977; Ishikawa et al., 2009). These data indicated that TDM per se triggers an important reaction to mycobacterial infection, which has traditionally been viewed as a correlate of protective immune responses, required to wall off infection. TDM also possesses adjuvant properties and is contained in the experimental adjuvant Ribi (Gavin et al., 2006). While these properties of TDM indicated that it acted as a pathogen-associated molecular pattern (PAMP), indicating microbial danger and alerting innate immune cells, the cord factor has also been recognized since so long as a virulence factor of mycobacteria (Figure 1). First, extraction of glycolipids with petroleum ether from the cell wall of MTB rendered the mycobacteria avirulent, due to efficient killing of the TDM-less mycobacteria inside macrophages (Indrigo et al., 2002, 2003). Second, when replacing this rough and crippling procedure with genetic ablation of TDM biosynthesis, the attenuated phenotype was confirmed in $\triangle \mathrm{fbpA}$ mutants lacking the mycolic acid transferase antigen 85A (Katti et al., 2008). In addition, these mutants triggered enhanced production of inflammatory cytokines and increased antigen presentation from infected macrophages (Kan-Sutton et al., 2009). Third, TDM coated onto beads is sufficient to delay phagosome maturation in macrophages (Axelrod et al., 2008), whereas the mutant MTB lacking TDM failed to block phagosome maturation (Katti et al., 2008). Together, the cord factor qualifies as a glycolipid effector molecule of pathogenic mycobacteria that reprograms macrophages for mycobacterial immune evasion and creation of a niche in the phagosome.

\section{TDM AS PAMP}

\section{A ROLE FOR TLR?}

Despite the decade-long knowledge of the inflammatory capacities of the cord factor, the mechanisms of immune cell activation by TDM were elusive until recently. As an abundant mycobacterial cell wall glycolipid with a chemical structure not found in vertebrate organisms, TDM a priori qualifies as a prototypical PAMP that may signal the presence of mycobacterial danger to innate immune cells. The phenocopying of inflammatory and granulomatous responses-induced by whole mycobacteria by TDM in animal models, and its long known property as an adjuvant eliciting cellular immune responses to co-administered protein antigen, support the notion of TDM being a PAMP. After the discovery of the toll-like receptor (TLR) family and several mycobacterial ligands that are recognized by TLR family members (ManLAM, $19 \mathrm{kDa}$ lipopeptide), the search for a pattern recognition receptor sensing TDM naturally first focused on a potential role of TLR. In Geisel et al. (2005) reported that TDM-coated beads stimulated TNF and IL-6 production by macrophages 


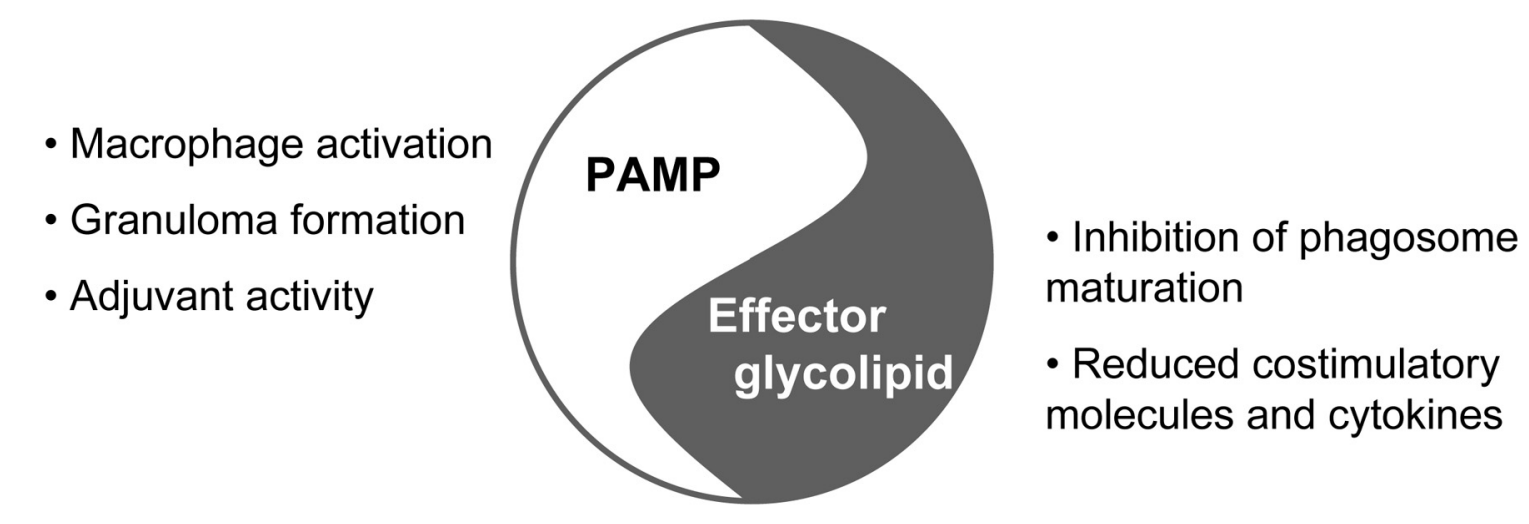

FIGURE 1 | TDM between PAMP and effector molecule.

in vitro, and that TDM in gel matrices injected s.c. into mice triggered massive leukocyte infiltration (Geisel et al., 2005). The role of TLR2 and TLR4, and of the adapter protein Myd88, was investigated exclusively for the in vivo leukocyte recruitment but not for the macrophage cytokine response in vitro. While TLR $2^{-/-}$and TLR4 ${ }^{-/}$mice had no defect in leukocyte recruitment to TDM gel matrices, Myd88-deficient mice showed a severe reduction, pointing to a possible role for other TLRs or of other Myd88utilizing receptors, e.g., for the cytokines IL-1 or IL-18. This initial report from the Russell lab was followed by a study reporting that murine macrophages deficient in TLR2 and in TLR4 had a strongly reduced response to TDM (Bowdish et al., 2009). In addition, the scavenger receptor MARCO was suggested to bind TDM in this study. Since scavenger receptors lack a cytoplasmic domain and are usually endocytic clearance receptors rather than activating PRR, a model was proposed where MARCO binding of TDM serves as a prerequisite for delivery to and activation of TLRs (Bowdish et al., 2009). In this context, a previous study is also of interest that investigated macrophages lacking Scavenger receptor A (SR-A) in vitro and observed an increased inflammatory response; importantly, these authors reported also that SR-A bound to the cord factor (Ozeki et al., 2006).

\section{TDM ACTIVATES MINCLE-Syk-Card9 SIGNALING}

Our own studies first aimed at determining a role of the TLR pathway for macrophage and DC activation-induced by TDM and its synthetic analog TDB. We observed that bone marrow derived macrophages from mice lacking Myd88 responded comparably to wild type cells (Werninghaus et al., 2009). In addition, the adjuvant activity of the cord factor analog TDB was preserved in mice deficient in TLR2, TLR3, TLR4, and TLR7 (Agger et al., 2008). This apparent lack of TLR-dependence led us to consider other PRR pathways. At this time, the C-type lectin receptor Dectin-1 (Brown, 2006) had just been described to activate macrophages via Syk-Card9 signaling (Gross et al., 2006). We employed macrophages from the respective KO mice to genetically define that macrophage activation by TDB and TDM requires Syk-Card9-Bcl10-Malt1 signaling, but is independent of Dectin-1 (Werninghaus et al., 2009). In contrast to Dectin-1 with its intracellular non-classical ITAM motif, other
CLR activate Syk via association with an adapter protein carrying the classical ITAM motif (Robinson et al., 2006). The requirement for the adapter protein FcRg but not Dap12 pointed to a certain set of C-type lectin receptors, including the family member Mincle (gene symbol: Clec4e) that was expressed inducibly in macrophages stimulated with the glycolipids. Mincle had been described in 2008 by Yamasaki et al. as sensor of necrosis that binds SAP130, a splicing factor released from dying cells (Yamasaki et al., 2008). Using independent lines of Mincle KO mice, Yamasaki et al. and Wells et al. demonstrated a function of Mincle in the recognition of fungal carbohydrates in the cell wall of Candida and Malassezia yeast (Wells et al., 2008; Yamasaki et al., 2009). A role for Mincle in recognition of mycobacteria was suggested by Yamasaki's group through experiments using a Mincle-reporter cell line. In a series of elegant experiments, they then identified the TDM from cell wall lipid fractionations as the active compound, showed direct binding of Mincle to TDM, and demonstrated that Mincle $^{-/-}$mice fail to produce the granulomatous response to TDM observed in the lungs of wild-type mice (Ishikawa et al., 2009). In parallel, experiments with Mincle-deficient mice in our lab showed that TDB and TDM do not activate macrophages from these mice anymore and that the Th1/Th17 adjuvant effect of TDB requires Mincle in vivo (Schoenen et al., 2010). Together, these studies provided solid evidence that Mincle is an essential receptor for the mycobacterial cord factor (Matsunaga and Moody, 2009) (Figure 2). Until now, however, the requirement for Mincle in TDM recognition has only been shown in the mouse system, and the possibility that the response to the cord factor in human macrophages is mediated by the same receptor and pathway remains to be proven experimentally (Lang et al., 2011).

\section{POTENTIAL FOR SYNERGY AND CROSS-REGULATION OF TLR AND MINCLE SIGNALING}

The reasons for the discordant results concerning the involvement of TLR-Myd88 signaling for TDM recognition between the Bowdish et al. (2009) and our studies (Werninghaus et al., 2009; Schoenen et al., 2010) are at present unclear. Differences in the type of macrophage used (bone marrow derived vs. resident peritoneal macrophages), culture conditions, and the presentation of 


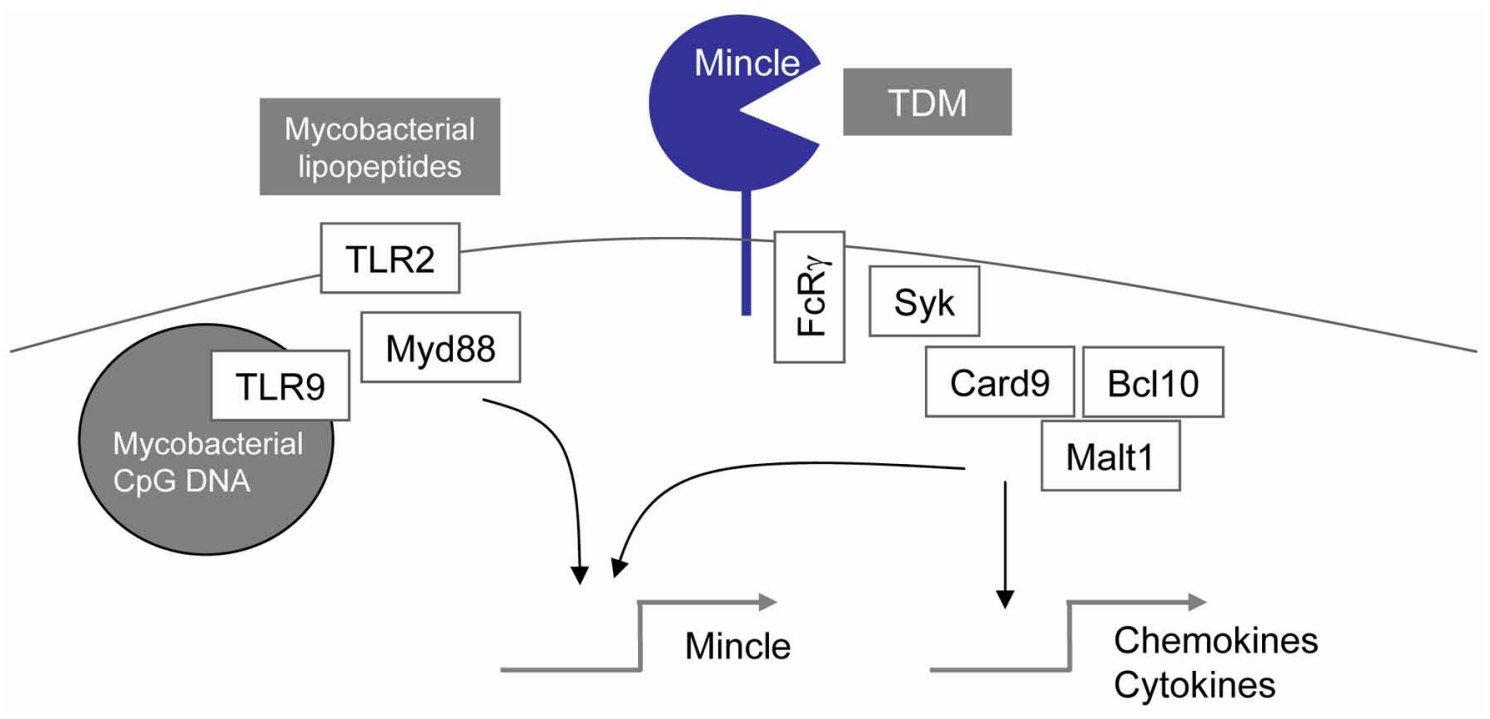

FIGURE 2 | TDM recognition and responses through regulated Mincle expression: possible synergy with TLR signals.

TDM (coated onto the cell culture dish vs. presented on beads) may be responsible. In general, TLR and CLR pathways can synergize to activate gene expression in macrophages. This has been clearly shown in the case of zymosan-triggered activation of macrophages through Dectin-1 and TLR2 (Dennehy et al., 2008, 2009). In the case of Mincle-mediated recognition of the cord factor, the inducible expression of the Mincle receptor following stimulation with TLR ligands (Matsumoto et al., 1999) suggests a mechanism of priming of macrophages for increased sensitivity to the CLR ligand TDM (Figure 2). The role of the MARCOTLR2-Myd88 pathway described by Bowdish et al. for TDM recognition (Bowdish et al., 2009) may therefore consist in licensing of macrophages for TDM responsiveness through induction of Mincle expression. A synergistic effect of Myd88 and Mincle for TDM responsiveness has very recently been shown for neutrophils by Lee et al. (2012): Myd88 $8^{-/}$neutrophils responded equally well with TNF release and adhesion to TDM as WT; in contrast, upon combined treatment with the TLR2 ligand Pam3CSK and TDM, synergistic induction was observed in WT but not Myd88-/- neutrophils, creating a significantly reduced response in Myd $88^{-/-}$vs. wild-type neutrophils that correlated with a lack of up-regulation of Mincle in the Myd88 ${ }^{-/}$cells (Lee et al., 2012).

\section{IS MINCLE REQUIRED FOR ANTI-MYCOBACTERIAL IMMUNITY? CONTRADICTORY ANSWERS FROM MOUSE INFECTION MODELS}

While the identification of Mincle as receptor for the cord factor solved a longstanding issue in TB research and provided a molecular basis for the adjuvant effect of TDM and TDB, the medically most important question remains whether Mincle and its associated pathway are required for anti-mycobacterial immunity, or may to the contrary be used by the pathogen to subvert immune responses.

\section{In vitro STUDIES WITH PHAGOCYTES}

The first steps taken to address the importance of the MincleSyk-Card9 pathway in the dealing of innate immune cells with infecting mycobacteria were in vitro infection experiments of macrophages with $\mathrm{M}$. bovis $B C G$ or MTB. These experiments showed a differential requirement for Mincle-signaling in the expression of several genes-induced by mycobacterial infection. Mincle-deficient macrophages expressed 10-fold less G-CSF and IL-6 in response to BCG (Schoenen et al., 2010). More recently, Behler et al. confirmed the essential role of Mincle for inflammatory cytokine and chemokine production in response to BCG in alveolar macrophages (Behler et al., 2012). Ishikawa et al. observed a slight reduction in the levels of several chemokines in $\mathrm{Mincle}^{-/-}$macrophages infected with MTB; this dependence on Mincle was strongly enlarged when macrophages from a Myd $88^{-/-}$background were used (Ishikawa et al., 2009). Thus, a picture emerges that in the absence of Mincle macrophages can still sense mycobacteria through recognition of PAMPs other than TDM, but the inflammatory response is compromised at least for a subset of response genes. To date no published data is available that has tested whether phagocytosis of mycobacteria is reduced or delayed in $\mathrm{Mincle}^{-/-}$macrophages.

\section{In vivo MYCOBACTERIAL INFECTION MODELS}

To date, three publications have reported on experimental mycobacterial infection in Mincle ${ }^{-/-}$mice (Behler et al., 2012; Heitmann et al., 2012; Lee et al., 2012) (see Table 1). Heitmann et al. used aerosol infection with MTB H37Rv and comprehensively analysed bacterial burden in lung and spleen, as well as the inflammatory and $\mathrm{T}$ cell responses in the lungs and draining lymph nodes at three different time points after infection. In addition, histopathological analysis of the lungs for the extent of infiltration, granulomatous response and immunocytochemistry for iNOS expression was performed. None of these readouts 
Table 1 | C-type lectin receptors in mycobacterial infection.

\begin{tabular}{|c|c|c|c|}
\hline & Dectin-1 & Mincle & Signr3 \\
\hline Mycobacterial ligand & Unknown & TDM & ManLAM \\
\hline Expression of CLR & Macrophages and DC & $\begin{array}{l}\text { Inducible in macrophages and DC; } \\
\text { High constitutive expression in } \\
\text { granulocytes }\end{array}$ & $\begin{array}{l}\text { Up-regulated in alveolar } \\
\text { macrophages during infection }\end{array}$ \\
\hline
\end{tabular}

Phenotype of knockout mouse in mycobacterial infection

References
Slightly reduced bacterial load in the lung: Unchanged adaptive immune response
Increased bacterial dissemination after BCG infection (Behler et al.,

2012);

Increased pulmonary load after

MTB Erdman (Lee et al., 2012);

Unchanged resistance after MTB

H37Rv (Heitmann et al., 2012)
Increased mycobacterial load early in infection ;

Intact adaptive immune responses showed a significant difference between $\mathrm{Mincle}^{-/-}$and WT control mice, leading to the conclusion that Mincle is not essential for the host response and protection against $\mathrm{TB}$ infection in mice. Low-dose aerosol infection with MTB is arguably the best mouse model for human TB; however, there are examples of knockout mice where the deletion of a gene did not cause a phenotype in the low dose model, but mice showed higher bacterial burden when challenged with a higher dose or by a different route (Reiling et al., 2002). To test for this possibility, Heitmann et al. also challenged Mincle ${ }^{-/-}$mice with a high dose of MTB H37Rv, again with no discernible difference in the bacterial load.

The second study reporting on the phenotype of Mincle ${ }^{-/-}$ mice in MTB is the paper by Lee et al., who focused their interest on the expression and function of Mincle in neutrophilic granulocytes during in vivo and in vitro encounter with TDM (Lee et al., 2012). Corroborating and extending the results obtained by Yamasaki's group (Ishikawa et al., 2009), they showed that Mincle $^{-/-}$mice fail to mount a granulomatous response to intravenously injected TDM and that Mincle expression on neutrophils is required for recruitment to TDM-coated beads injected subcutaneously. They further demonstrate that neutrophils attach to plate-bound TDM in vitro and respond in a Mincle-dependent manner with up-regulation of integrins and production of reactive oxygen species. These results are interesting because they draw attention to neutrophils which express higher levels of Mincle than macrophages and can immediately respond to TDM. Lee et al. also demonstrate that depletion of neutrophils with an anti-Ly6G antibody reduced the initial granulomatous response and the levels of IL- 6 and MCP-1 in the lungs of TDM-injected mice. The analysis of MTB infection in the Mincle $^{-/-}$mice in the study by Lee et al. consists of one low-dose aerosol infection experiment where bacterial loads in the lungs were determined at two time-points after infection, showing a significant but moderate increase $\left(0.5 \log _{10}\right)$. The increased mycobacterial numbers in the $\mathrm{Mincle}^{-/-}$mice were paralleled by a stronger expression of inflammatory genes in the lungs. Given the expression and function of Mincle in granulocytes described in the same paper, it would be interesting to know whether neutrophil accumulation, integrin expression and cytokine production in the lungs is particularly affected in the mice infected in the Lee et al. study. The higher expression of inflammatory genes in Mincle $^{-/-}$lungs is difficult to interpret because it could be entirely due to the increased mycobacterial load generating more PAMPs for activation of receptors other than Mincle. One can only speculate at which time after infection Mincle $^{-/-}$mice start to have higher MTB burden in the lung; one possibility is that a lack of recruitment and proper activation of neutrophils early after infection gives MTB the ability to survive and grow better than in WT mice.

In contrast, the third study, reported by Behler et al., used the vaccine strain $M$. bovis BCG in intratracheal and intravenous infection models (Behler et al., 2012). These authors first characterized the expression of Mincle on alveolar macrophages following exposure to BCG in vivo, and observed a strong upregulation of receptor levels on the cell surface. This is consistent with previous observations in vitro at the mRNA and protein level (Yamasaki et al., 2008; Werninghaus et al., 2009; Schoenen et al., 2010), and importantly it first demonstrates that this process occurs during mycobacterial encounter in vivo, implying that Mincle induction by mycobacterial signals could serve to bolster recognition and defense against spread of mycobacteria or secondary challenge by subsequent exposure. In fact, this is exactly what the authors found in their further experiments: when mice were infected twice with BCG separated by 14 days, the inflammatory response to BCG in the airways and in alveolar macrophages strongly increased in a Mincle-dependent fashion. More importantly, the priming infection increased the Mincle-dependence of BCG-growth restriction, showing that the up-regulation of Mincle during the priming period indeed led to better innate control of intruding mycobacteria by alveolar macrophages. Another interesting effect of Mincle-deficiency in this study is the stronger increase in the bacterial burden in the draining lymph nodes and the spleen, compared to the lungs after intratracheal BCG infection (Behler et al., 2012). Dissemination of BCG after airway infection requires time until pulmonary mycobacteria have sufficiently expanded, allowing the quite slow induction of Mincle on alveolar macrophages (and also on other recruited cells, e.g., neutrophils) to generate the higher capacity of BCG recognition and 
to mount a better response leading to containment of infection at the local site.

Taken together, these recent studies on the role of Mincle in the host response to mycobacterial infection yielded remarkably different results, raising the question for possible reasons that underlie the discrepant findings.

First, the three studies used different strains of $M$. tuberculosis (H37Rv in the Heitmann et al., Erdman in the Lee et al. study) or employed the vaccine strain M. bovis BCG (Behler et al.). Although all these strains have abundant TDM in the cell wall, they may differ in the length of mycolic acid chains, modifications and the mixture of other glycolipids present in the cell wall, which may account for the difference in the requirement for Mincle. Of interest, in vitro infection experiments of macrophages showed a stronger dependence of the NO production and of G-CSF secretion on Mincle when BCG was used compared to MTB H37Rv (Heitmann et al., 2012), which appears consistent with the larger role of Mincle in BCG vs. MTB H37Rv infection.

The relative amount of TDM present in the mycobacterial cell may also be affected by glycolipid exchange through the action of mycolyltransferases. Increased levels of glucose, as they are encountered by the mycobacteria in the cellular growth environment, can lead to a shift in glycolipid synthesis from TDM to glucose monomycolate (GMM) in an Ag85A-dependent manner (Matsunaga et al., 2008). GMM is a ligand for CD1b in humans (Moody et al., 1997), it has not been formally tested for binding to and activation of Mincle.

In principle, another variable can be introduced by changes in the culture conditions used to grow the mycobacteria before infection. For example, the commonly used inclusion of detergents like Tween in the mycobacterial broth to prevent clumping has been shown to drastically modify the presence of a polysaccharide capsular structure (Sani et al., 2010) and may also wash out TDM from the mycobacterial cell wall. Indeed, the inclusion of Tween in ELISA wash buffers can even remove coated glycolipids from microtiter plate plastic (Julian et al., 2001) and drastically reduce the detection of TDM-specific antibodies from the sera of TB patients (Traunmuller et al., 2005). It is therefore conceivable that inter-strain differences and subtle changes in the culture conditions used can significantly affect the composition of the mycobacterial cell wall and capsule, thereby affecting the interaction with Mincle and other pattern recognition receptors and changing the outcome of infection.

Finally, the hygiene status of mouse colonies can be expected to alter the basal levels of innate immune activation in general and of Mincle expression levels on phagocytes in particular through effects of TLR- and CLR-dependent PAMPs.

\section{ROLE OF OTHER C-TYPE LECTIN RECEPTORS IN ANTI-MYCOBACTERIAL IMMUNITY}

The adapter protein Card9 is expressed in myeloid cells and was initially identified as crucial for transducing the signal of the C-type lectin receptor Dectin-1 to NFkB activation (Gross et al., 2006). Card9 is down-stream of Syk and becomes phosphorylated by PKC delta (Strasser et al., 2012). Card9 is not only required for Dectin-1 signaling, but is also the central adapter for DAP12- and
FcR $\gamma$-chain-coupled C-type lectin receptors like Mincle, Dectin-2 and Clec5a (Hara et al., 2007; Yamasaki et al., 2008; Werninghaus et al., 2009). Dorhoi et al. showed that the absence of Card9 renders mice exquisitely sensitive to infection with MTB (Dorhoi et al., 2010). Following aerosol infection, Card $9^{-/}$mice develop high mycobacterial burden in the lungs and die within 4-6 weeks. Card $9^{-/}$macrophages normally phagocytosed and killed MTB in vitro, but were impaired in the production of inflammatory cytokines and chemokines. In vivo, Card $9^{-/-}$mice had a striking increase in neutrophil infiltration in the lung that was linked to tissue destruction and death of the mice by depletion experiments (Dorhoi et al., 2010). This essential role of Card9 in anti-mycobacterial defense, together with the relative lack of a phenotype in Mincle-deficient mice, poses the question which other C-type lectin receptors signaling through Card9 are triggered during MTB infection (see Table $\mathbf{1}$ ).

Dectin-1 has been implicated by several labs in the response to mycobacteria, but the nature of the ligand has remained unknown to date. Blocking Dectin-1 with antibodies or laminarin reduced cytokine responses to M. bovis BCG or MTB (Yadav and Schorey, 2006; Rothfuchs et al., 2007). In addition, a reduced uptake of $M$. abscessus and impaired ROS production was reported when Dectin-1 was blocked in mouse macrophages (Shin et al., 2008). To test the contribution of Dectin-1-mediated recognition of mycobacteria in vivo, Brown and colleagues challenged Dectin-1 KO mice with virulent MTB. The mycobacterial load in the lungs was unexpectedly slightly reduced in Dectin-1 KO mice, but there were no significant differences in lung pathology or adaptive immune responses (Marakalala et al., 2011). Thus, Dectin-1 appears to play a rather redundant role for protection against MTB.

The human C-type lectin DC-SIGN has received considerable attention in the mycobacterial immunity field when it was discovered that binding of mycobacterial Man-LAM to DC-SIGN on human macrophages down-regulates costimulatory molecules and inflammatory responses, presumably via induction of IL-10 production (Geijtenbeek et al., 2003). There are several murine homologues of human DC-SIGN in a cluster of seven genes and one pseudogene (Tanne et al., 2009). Based on the binding to glycans with high mannose content and fucose-containing oligosaccharides, mouse SIGNR3 is the best candidate as ortholog for human DC-SIGN. Tanne et al. created knockout mice for Signr1, Signr3 and Signr5, and determined their phenotype in TB infection (Tanne et al., 2009). The mycobacterial load in the lungs of Signr3-deficient mice was clearly increased, whereas knockout mice for Signr1 and Signr5 were indistinguishable from wildtype controls. However, the development of adaptive immune response to TB was not compromised in the absence of SIGNR3 and the mice did not die at higher rates compared to controls of TB. Of note, SIGNR3 was up-regulated on lung phagocytic cells during infection, and the increased mycobacterial load was specific for the lung while no difference between WT and Signr3-/mice was seen in the spleen. SIGNR3 binding of ManLAM and whole mycobacteria induces production of proinflammatory cytokines through activation of Syk and the kinase Raf1 (Tanne et al., 2009). Although the requirement for Card9 in the activation of macrophages and DC in response to ligation of SIGNR3 
has not been formally demonstrated, it appears very likely. Hence, a defect in the signaling of SIGRNR3 may contribute to the strong phenotype of the Card $9^{-/-}$mice in tuberculosis, although it appears to be essential for anti-mycobacterial resistance only during the early innate phase of infection.

Given the large number of CLR that can be expressed in myeloid cells, additional players must be expected to contribute to recognition of mycobacterial ligands and to macrophage activation and anti-mycobacterial defense via Card9-dependent mechanisms. Another interesting candidate to look at is Dectin-2; of note a Dectin-2-Fc fusion protein binds to the surface of mycobacteria (McGreal et al., 2006). Similar to Mincle, Dectin-2 utilizes the $\mathrm{FcR} \gamma$ chain as adapter molecule. Interestingly, FcR $\gamma$ chain-deficient mice develop increased mycobacterial burden, enhanced immunpathology and earlier death when challenged with MTB (Maglione et al., 2008). While Chan and colleagues

\section{REFERENCES}

Agger, E. M., Rosenkrands, I., Hansen, J., Brahimi, K., Vandahl, B. S., Aagaard, C., et al. (2008). Cationic liposomes formulated with synthetic mycobacterial cordfactor (CAF01): a versatile adjuvant for vaccines with different immunological requirements. PLoS ONE 3:e3116. doi: 10.1371/journal.pone.0003116

Axelrod, S., Oschkinat, H., Enders, J., Schlegel, B., Brinkmann, V., Kaufmann, S. H., et al. (2008). Delay of phagosome maturation by a mycobacterial lipid is reversed by nitric oxide. Cell. Microbiol. 10, 1530-1545.

Behler, F., Steinwede, K., Balboa, L., Ueberberg, B., Maus, R., Kirchhof, G., et al. (2012). Role of Mincle in alveolar macrophage-dependent innate immunity against mycobacterial infections in mice. J. Immunol. 189, 3121-3129.

Bowdish, D. M., Sakamoto, K., Kim, M. J., Kroos, M., Mukhopadhyay, S., Leifer, C. A., et al. (2009). MARCO, TLR2, and CD14 are required for macrophage cytokine responses to Mycobacterial trehalose dimycolate and Mycobacterium tuberculosis. PLoS Pathog. 5:e1000474. doi: 10.1371/journal.ppat.1000474

Brown, G. D. (2006). Dectin-1, a signalling non-TLR patternrecognition receptor. Nat. Rev. Immunol. 6, 33-43.

Dennehy, K. M., Ferwerda, G., FaroTrindade, I., Pyz, E., Willment, J. A., Taylor, P. R., et al. (2008). Syk kinase is required for collaborative cytokine production induced through Dectin-1 and Toll-like receptors. Eur. J. Immunol. 38, 500-506.

Dennehy, K. M., Willment, J. A., Williams, D. L., and Brown, G. D.
(2009). Reciprocal regulation of IL-23 and IL-12 following coactivation of Dectin-1 and TLR signaling pathways. Eur. J. Immunol. 39, 1379-1386.

Dorhoi, A., Desel, C., Yeremeev, V., Pradl, L., Brinkmann, V., Mollenkopf, H. J., et al. (2010). The adaptor molecule CARD9 is essential for tuberculosis control. J. Exp. Med. 207, 777-792.

Gavin, A. L., Hoebe, K., Duong, B., Ota, T., Martin, C., Beutler, B., et al. (2006). Adjuvant-enhanced antibody responses in the absence of toll-like receptor signaling. Science 314, 1936-1938.

Geijtenbeek, T. B., Van Vliet, S. J., Koppel, E. A., Sanchez-Hernandez, M., Vandenbroucke-Grauls, C. M., Appelmelk, B., et al. (2003). Mycobacteria target DC-SIGN to suppress dendritic cell function. J. Exp. Med. 197, 7-17.

Geisel, R. E., Sakamoto, K., Russell, D. G., and Rhoades, E. R. (2005). In vivo activity of released cell wall lipids of Mycobacterium bovis bacillus Calmette-Guerin is due principally to trehalose mycolates. J. Immunol. 174, 5007-5015.

Gross, O., Gewies, A., Finger, K., Schafer, M., Sparwasser, T., Peschel, C., et al. (2006). Card9 controls a non-TLR signalling pathway for innate anti-fungal immunity. Nature 442, 651-656.

Hara, H., Ishihara, C., Takeuchi, A., Imanishi, T., Xue, L., Morris, S. W., et al. (2007). The adaptor protein CARD9 is essential for the activation of myeloid cells through ITAMassociated and toll-like receptors. Nat. Immunol. 8, 619-629.

Heitmann, L., Schoenen, H., Ehlers, S., Lang, R., and Holscher, C. (2012). Mincle is not essential for controlling

discuss their findings exclusively in the context of $\mathrm{FcR} \gamma$ chain's role as adapter of activating $\mathrm{Fc}$ gamma receptors mediating antibody effector function, the dual use of the adapter in recruiting Syk also to several CLR family members could also indicate that the phenotype is at least in part due to a lack of signaling by Mincle, Dectin- 2 and other CLRs. Likewise, the activation of the FcR $\gamma$-Syk-Card9 signaling cascade by Fc gamma receptors would allow a contribution of antibody-mediated effects to Card9-dependent protection in TB infection, a possibility that has not been explored to date.

\section{ACKNOWLEDGMENTS}

Research in the Lang lab on innate recognition and adjuvanticity of mycobacterial glycolipids has been funded by Deutsche Forschungsgemeinschaft (SFB 796, B6) and European Union (FP6 TBVAC and FP7 NEWTBVAC).

Mycobacterium tuberculosis infection. Immunobiology. doi: 10.1016/ j.imbio.2012.06.005. [Epub ahead of print].

Hunter, R. L., Olsen, M. R., Jagannath, C., and Actor, J. K. (2006). Multiple roles of cord factor in the pathogenesis of primary, secondary, and cavitary tuberculosis, including a revised description of the pathology of secondary disease. Ann. Clin. Lab. Sci. 36, 371-386.

Indrigo, J., Hunter, R. L. Jr., and Actor, J. K. (2002). Influence of trehalose 6, 6' -dimycolate (TDM) during mycobacterial infection of bone marrow macrophages. Microbiology 148, 1991-1998.

Indrigo, J., Hunter, R. L. Jr., and Actor, J. K. (2003). Cord factor trehalose 6 , 6 -dimycolate (TDM) mediates trafficking events during mycobacterial infection of murine macrophages. Microbiology 149, 2049-2059.

Ishikawa, E., Ishikawa, T., Morita, Y. S., Toyonaga, K., Yamada, H., Takeuchi, O., et al. (2009). Direct recognition of the mycobacterial glycolipid, trehalose dimycolate, by C-type lectin Mincle. J. Exp. Med. 206, 2879-2888.

Julian, E., Cama, M., Martinez, P., and Luquin, M. (2001). An ELISA for five glycolipids from the cell wall of Mycobacterium tuberculosis: Tween 20 interference in the assay. J. Immunol. Methods 251, 21-30.

Kan-Sutton, C., Jagannath, C., and Hunter, R. L. Jr. (2009). Trehalose $6,6^{\prime}$-dimycolate on the surface of Mycobacterium tuberculosis modulates surface marker expression for antigen presentation and costimulation in murine macrophages. Microbes Infect. 11, 40-48.

Katti, M. K., Dai, G., Armitige, L. Y., Rivera Marrero, C., Daniel, S., Singh, C. R., et al. (2008).
The Delta fbpA mutant derived from Mycobacterium tuberculosis $\mathrm{H} 37 \mathrm{Rv}$ has an enhanced susceptibility to intracellular antimicrobial oxidative mechanisms, undergoes limited phagosome maturation and activates macrophages and dendritic cells. Cell. Microbiol. 10, 1286-1303.

Lang, R., Schoenen, H., and Desel, C. (2011). Targeting Syk-Card9activating C-type lectin receptors by vaccine adjuvants: findings, implications and open questions. Immunobiology 216, 1184-1191.

Lee, W. B., Kang, J. S., Yan, J. J., Lee, M. S., Jeon, B. Y., Cho, S. N., et al. (2012). Neutrophils promote Mycobacterial trehalose dimycolate-induced lung inflammation via the Mincle pathway. PLoS Pathog. 8:e1002614. doi: 10.1371/journal.ppat.1002614

Maglione, P. J., Xu, J., Casadevall, A., and Chan, J. (2008). Fc gamma receptors regulate immune activation and susceptibility during Mycobacterium tuberculosis infection. J. Immunol. 180, 3329-3338.

Marakalala, M. J., Guler, R., Matika, L., Murray, G., Jacobs, M., Brombacher, F., et al. (2011). The Syk/CARD9-coupled receptor Dectin-1 is not required for host resistance to Mycobacterium tuberculosis in mice. Microbes Infect. 13, 198-201.

Matsumoto, M., Tanaka, T., Kaisho, T., Sanjo, H., Copeland, N. G., Gilbert, D. J., et al. (1999). A novel LPS-inducible C-type lectin is a transcriptional target of NF-IL6 in macrophages. J. Immunol. 163, 5039-5048.

Matsunaga, I., and Moody, D. B. (2009). Mincle is a long sought receptor for mycobacterial cord factor. J. Exp. Med. 206, 2865-2868. 
Matsunaga, I., Naka, T., Talekar, R. S., McConnell, M. J., Katoh, K., Nakao, H., et al. (2008). Mycolyltransferase-mediated glycolipid exchange in Mycobacteria. J. Biol. Chem. 283, 28835-28841.

McGreal, E. P., Rosas, M., Brown, G. D., Zamze, S., Wong, S. Y., Gordon, S., et al. (2006). The carbohydraterecognition domain of Dectin-2 is a C-type lectin with specificity for high mannose. Glycobiology 16, 422-430.

Moody, D. B., Reinhold, B. B., Guy, M. R., Beckman, E. M., Frederique, D. E., Furlong, S. T., et al. (1997). Structural requirements for glycolipid antigen recognition by CD1b-restricted T cells. Science 278, 283-286.

Ozeki, Y., Tsutsui, H., Kawada, N., Suzuki, H., Kataoka, M., Kodama, T., et al. (2006). Macrophage scavenger receptor down-regulates mycobacterial cord factor-induced proinflammatory cytokine production by alveolar and hepatic macrophages. Microb. Pathog. 40, 171-176.

Reiling, N., Holscher, C., Fehrenbach, A., Kroger, S., Kirschning, C. J., Goyert, S., et al. (2002). Cutting edge: toll-like receptor (TLR)2- and TLR4-mediated pathogen recognition in resistance to airborne infection with Mycobacterium tuberculosis. J. Immunol. 169, 3480-3484.

Robinson, M. J., Sancho, D., Slack, E. C., Leibundgut-Landmann, S., and Sousa, C. R. (2006). Myeloid C-type lectins in innate immunity. Nat. Immunol. 7, 1258-1265.

Rothfuchs, A. G., Bafica, A., Feng, C. G., Egen, J. G., Williams, D. L.,
Brown, G. D., et al. (2007). Dectin-1 interaction with Mycobacterium tuberculosis leads to enhanced IL-12p40 production by splenic dendritic cells. J. Immunol. 179, 3463-3471.

Sani, M., Houben, E. N., Geurtsen, J., Pierson, J., de Punder, K., van Zon, M., et al. (2010). Direct visualization by cryo-EM of the mycobacterial capsular layer: a labile structure containing ESX-1-secreted proteins. PLoS Pathog. 6:e1000794. doi: 10.1371/ journal.ppat.1000794

Schoenen, H., Bodendorfer, B. Hitchens, K., Manzanero, S., Werninghaus, K., Nimmerjahn, F., et al. (2010). Cutting edge: Mincle is essential for recognition and adjuvanticity of the mycobacterial cord factor and its synthetic analog trehalose-dibehenate. J. Immunol. 184, 2756-2760.

Shin, D. M., Yang, C. S., Yuk, J. M., Lee, J. Y., Kim, K. H., Shin, S. J., et al. (2008). Mycobacterium abscessus activates the macrophage innate immune response via a physical and functional interaction between TLR2 and dectin-1. Cell. Microbiol. 10, 1608-1621.

Strasser, D., Neumann, K., Bergmann, H., Marakalala, M. J., Guler, R., Rojowska, A., et al. (2012). Syk kinase-coupled C-type lectin receptors engage protein kinase $\mathrm{C}$-sigma to elicit Card9 adaptor-mediated innate immunity. Immunity 36, 32-42.

Tanne, A., Ma, B., Boudou, F., Tailleux, L., Botella, H., Badell, E., et al. (2009). A murine DC-SIGN homologue contributes to early host defense against Mycobacterium tuberculosis. J. Exp. Med. 206, 2205-2220.

Traunmuller, F., Haslinger, I., Lagler, H., Wolfgang, G., Zeitlinger, M. A., and Abdel Salam, H. A. (2005). Influence of the washing buffer composition on the sensitivity of an enzyme-linked immunosorbent assay using mycobacterial glycolipids as capture antigens. J. Immunoassay Immunochem. 26, 179-188.

Wells, C. A., Salvage-Jones, J. A., Li, X., Hitchens, K., Butcher, S., Murray, R. Z., et al. (2008). The macrophageinducible C-type lectin, mincle, is an essential component of the innate immune response to Candida albicans. J. Immunol. 180, 7404-7413.

Werninghaus, K., Babiak, A., Gross, O. Holscher, C., Dietrich, H., Agger, E. M., et al. (2009). Adjuvanticity of a synthetic cord factor analogue for subunit Mycobacterium tuberculosis vaccination requires FcRgamma-Syk-Card9-dependent innate immune activation. J. Exp. Med. 206, 89-97.

Yadav, M., and Schorey, J. S. (2006). The beta-glucan receptor dectin-1 functions together with TLR2 to mediate macrophage activation by mycobacteria. Blood 108 , 3168-3175.

Yamasaki, S., Ishikawa, E., Sakuma, M., Hara, H., Ogata, K., and Saito, T. (2008). Mincle is an ITAMcoupled activating receptor that senses damaged cells. Nat. Immunol. 9, 1179-1188.

Yamasaki, S., Matsumoto, M., Takeuchi, O., Matsuzawa, T.,
Ishikawa, E., Sakuma, M., et al. (2009). C-type lectin Mincle is an activating receptor for pathogenic fungus, Malassezia. Proc. Natl. Acad. Sci. U.S.A. 106, 1897-1902.

Yarkoni, E., and Rapp, H. J. (1977). Granuloma formation in lungs of mice after intravenous administration of emulsified trehalose- 6 , 6 '-dimycolate (cord factor): reaction intensity depends on size distribution of the oil droplets. Infect. Immun. 18, 552-554.

Conflict of Interest Statement: The author declares that the research was conducted in the absence of any commercial or financial relationships that could be construed as a potential conflict of interest.

Received: 31 October 2012; paper pending published: 17 December 2012; accepted: 04 January 2013; published online: 24 January 2013.

Citation: Lang $R$ (2013) Recognition of the mycobacterial cord factor by Mincle: relevance for granuloma formation and resistance to tuberculosis. Front. Immun. 4:5. doi: 10.3389/fimmu.2013.00005

This article was submitted to Frontiers in Inflammation, a specialty of Frontiers in Immunology.

Copyright (c) 2013 Lang. This is an open-access article distributed under the terms of the Creative Commons Attribution License, which permits use, distribution and reproduction in other forums, provided the original authors and source are credited and subject to any copyright notices concerning any third-party graphics etc. 